\title{
A common fixed point theorem for a commuting family of nonexpansive mappings one of which is multivalued
}

\author{
Narawadee Nanan ${ }^{1}$ and Sompong Dhompongsa ${ }^{1,2^{*}}$
}

* Correspondence:
sompongd@chiangmai.ac.th
${ }^{1}$ Department of Mathematics,
Faculty of Science, Chiang Mai
University, Chiang Mai 50200,
Thailand
Full list of author information is
available at the end of the article

* Correspondence: sompongd@chiangmaiacth Faculty of Science, Chiang Mai University, Chiang Mai 50200, Full list of author information is available at the end of the article

\begin{abstract}
Bruck [Pac. J. Math. 53, 59-71 1974 Theorem 1] proved that for a nonempty closed convex subset $E$ of a Banach space $X$, if $E$ is weakly compact or bounded and separable and suppose that $E$ has both (FPP) and (CFPP), then for any commuting family $S$ of nonexpansive self-mappings of $E$, the set $F(S)$ of common fixed points of $S$ is a nonempty nonexpansive retract of $E$. In this paper, we extend the above result when one of its elements in $S$ is multivalued. The result extends previously known results (on common fixed points of a pair of single valued and multivalued commuting mappings) to infinite number of mappings and to a wider class of spaces. 2000 Mathematics Subject Classification: 47H09; 47H10
\end{abstract}

Keywords: Common fixed point, Nonexpansive retract, Property (D), Kirk-Massa condition

\section{Introduction}

For a pair $(t, T)$ of nonexpansive mappings $t: E \rightarrow E$ and $T: E \rightarrow 2^{X}$ defined on a bounded closed and convex subset $E$ of a convex metric space or a Banach space $X$, we are interested in finding a common fixed point of $t$ and $T$. In [1], Dhompongsa et al. obtained a result for the $\mathrm{CAT}(0)$ space setting:

Theorem 1.1. [[1], Theorem 4.1] Let E be a nonempty bounded closed and convex subset of a complete CAT(O) space $X$, and let $t: E \rightarrow E$ and $T: E \rightarrow 2^{X}$ be nonexpansive mappings with $T(x)$ a nonempty compact convex subset of $X$. Assume that for some $p \in \operatorname{Fix}(t)$,

$$
\alpha p \oplus(1-\alpha) T x \text { is convex for } x \in E, \alpha \in[0,1] .
$$

If $t$ and $T$ are commuting, then $\operatorname{Fix}(t) \cap \operatorname{Fix}(T) \neq \varnothing$.

Shahzad and Markin [2] improved Theorem 1.1 by removing the assumption that the nonexpansive multivalued mapping $T$ in that theorem has a convex-valued contractive approximation. They also noted that Theorem 1.1 needs the additional assumption that $T(\cdot) \cap E \neq \varnothing$ for that result to be valid.

Theorem 1.2. [[2], Theorem 4.2] Let $X$ be a complete CAT(0) space, and E a bounded closed and convex subset of $X$. Assume $t: E \rightarrow E$ and $T: E \rightarrow 2^{X}$ are nonexpansive mappings with $T(x)$ a compact convex subset of $X$ and $T(x) \cap E \neq \varnothing$ for each $x$ $\in$ E. If the mappings $t$ and $T$ commute, then $\operatorname{Fix}(t) \cap \operatorname{Fix}(T) \neq \varnothing$. 
Dhompongsa et al. [3] extended Theorem 1.1 to uniform convex Banach spaces.

Theorem 1.3. [[3], Theorem 4.2] Let E be a nonempty bounded closed and convex subset of a uniform convex Banach space X. Assume $t: E \rightarrow E$ and $T: E \rightarrow 2^{E}$ are nonexpansive mappings with $T(x)$ a nonempty compact convex subset of $E$. If $t$ and $T$ are commuting, then $\operatorname{Fix}(t) \cap \operatorname{Fix}(T) \neq \varnothing$.

The result has been improved, generalized, and extended under various assumptions. See for examples, [[4], Theorem 3.3], [[5], Theorem 3.4], [[6], Theorem 3.9], [[7], Theorem 4.7], [[8], Theorem 5.3], [[9], Theorem 5.2], [[10], Theorem 3.5], [[11], Theorem 4.2], [[12], Theorem 3.8], [[13], Theorem 3.1].

Recall that a bounded closed and convex subset $E$ of a Banach space $X$ has the fixed point property for nonexpansive mappings (FPP) (respectively, for multivalued nonexpansive mappings (MFPP)) if every nonexpansive mapping of $E$ into $E$ has a fixed point (respectively, every nonexpansive mapping of $E$ into $2^{E}$ with compact convex values has a fixed point). The following concepts and result were introduced and proved by Bruck $[14,15]$. For a bounded closed and convex subset $E$ of a Banach space $X$, a mapping $t: E \rightarrow X$ is said to satisfy the conditional fixed point property (CFP) if either $t$ has no fixed points, or $t$ has a fixed point in each nonempty bounded closed convex set that leaves $t$ invariant. A set $E$ is said to have the hereditary fixed point property for nonexpansive mappings (HFPP) if every nonempty bounded closed convex subset of $E$ has the fixed point property for nonexpansive mappings; $E$ is said to have the conditional fixed point property for nonexpansive mappings (CFPP) if every nonexpansive $t: E \rightarrow E$ satisfies (CFP).

Theorem 1.4. [[15], Theorem 1] Let E be a nonempty closed convex subset of a Banach space X. Suppose E is weakly compact or bounded and separable. Suppose E has both (FPP) and (CFPP). Then for any commuting family $S$ of nonexpansive selfmappings of $E$, the set $F(S)$ of common fixed points of $S$ is a nonempty nonexpansive retract of $E$.

The object of this paper is to extend Theorems 1.3 and 1.4 for a commuting family $S$ of nonexpansive mappings one of which is multivalued. As consequences,

(i) Theorem 1.3 is extended to a bigger class of Banach spaces while a class of mappings is no longer finite;

(ii) Theroem 1.4 is extended so that one of its members in $S$ can be multivalued.

\section{Preliminaries}

Let $E$ be a nonempty subset of a Banach space $X$. A mapping $t: E \rightarrow X$ is said to be nonexpansive if

$$
\|t x-t y\| \leq\|x-y\|, x, y \in E .
$$

The set of fixed points of $t$ will be denoted by Fix $(t):=\{x \in E: t x=x\}$. A subset $C$ of $E$ is said to be $t$-invariant if $t(C) \subset C$. As usual, $B(x, \varepsilon)=\{y \in X:|| x-y||<\varepsilon\}$ stands for an open ball. For a subset $A$ and $\varepsilon>0$, the $\varepsilon$-neighborhood of $A$ is defined as

$$
B_{\varepsilon}(A)=\{y \in X:\|x-y\|<\varepsilon \text {, for some } x \in A\}=\bigcup_{x \in A} B(x, \varepsilon) .
$$

Note that if $A$ is convex, then $B_{\varepsilon}(A)$ is also convex. We write $\bar{A}$ for the closure of $A$. 
We shall denote by $2^{E}$ the family of all subsets of $E, C B(E)$ the family of all nonempty closed bounded subsets of $E$ and denote by $K C(E)$ the family of all nonempty compact convex subsets of $E$. Let $H(\cdot, \cdot)$ be the Hausdorff distance defined on $C B(X)$, i.e.,

$$
H(A, B):=\max \left\{\sup _{a \in A} \operatorname{dist}(a, B), \sup _{b \in B} \operatorname{dist}(b, A)\right\}, A, B \in C B(X),
$$

where $\operatorname{dist}(a, B):=\inf \{\|a-b\|: b \in B\}$ is the distance from the point $a$ to the subset $B$.

A multivalued mapping $T: E \rightarrow C B(X)$ is said to be nonexpansive if

$$
H(T x, T y) \leq\|x-y\| \text { for all } x, y \in E .
$$

$T$ is said to be upper semi-continuous if for each $x_{0} \in E$, for each neighborhood $U$ of $T x_{0}$, there exists a neighborhood $V$ of $x_{0}$ such that $T x \subset U$ for each $x \in V$. Clearly, every upper semi-continuous mapping $T$ has a closed graph, i.e., for each sequence $\left\{x_{n}\right\} \subset E$ converging to $x_{0} \in E$, for each $y_{n} \in T x_{n}$ with $y_{n} \rightarrow y_{0}$, one has $y_{0} \in T x_{0}$. Fix $(T)$ is the set of fixed points of $T$, i.e., $\operatorname{Fix}(T):=\{x \in E: x \in T x\}$. A subset $C$ of $E$ is said to be $T$-invariant if $T x \cap C \neq \varnothing$ for all $x \in C$. For $\lambda \in(0,1)$, we say that a multivalued mapping $T: E \rightarrow C B(X)$ satisfies condition $\left(C_{\lambda}\right)$ if $\lambda \operatorname{dist}(x, T x) \leq\|x-y\|$ implies $H(T x, T y) \leq\|x-y\|$ for $x, y \in E$. The following example shows that a mapping $T$ satisfying condition $\left(C_{\lambda}\right)$ for some $\lambda \in(0,1)$ can be discontinuous:

Let $\lambda \in(0,1)$ and $a=\frac{2(\lambda+1)}{\lambda(\lambda+2)}$. Define a mapping $T:\left[0, \frac{2}{\lambda}\right] \rightarrow K C\left(\left[0, \frac{2}{\lambda}\right]\right)$ by

$$
T x=\left(\begin{array}{ll}
\left\{\frac{x}{2}\right\} & \text { if } x \neq \frac{2}{\lambda} \\
{\left[\frac{1}{\lambda}, a\right]} & \text { if } x=\frac{2}{\lambda} .
\end{array}\right.
$$

Clearly, $\frac{1}{\lambda}<a<\frac{2}{\lambda}$ and $T$ is nonexpansive on [0, $\frac{2}{\lambda}$ ). Thus, we only verify that, for $\lambda \operatorname{dist}(x, T x) \leq\left\|x-\frac{2}{\lambda}\right\| \Rightarrow H\left(T x, T \frac{2}{\lambda}\right) \leq\left\|x-\frac{2}{\lambda}\right\|$,

$$
\lambda \operatorname{dist}(x, T x) \leq\left\|x-\frac{2}{\lambda}\right\| \Rightarrow H\left(T x, T \frac{2}{\lambda}\right) \leq\left\|x-\frac{2}{\lambda}\right\|
$$

and

$$
\lambda \operatorname{dist}\left(\frac{2}{\lambda}, T \frac{2}{\lambda}\right) \leq\left\|\frac{2}{\lambda}-x\right\| \Rightarrow H\left(T \frac{2}{\lambda}, T x\right) \leq\left\|\frac{2}{\lambda}-x\right\| .
$$

If $\lambda \operatorname{dist}(x, T x) \leq\left\|x-\frac{2}{\lambda}\right\|$, then $x \leq \frac{4}{\lambda(\lambda+2)}$ and

$$
H\left(T x, T \frac{2}{\lambda}\right)=a-\frac{x}{2} \leq \frac{2}{\lambda}-x=\left\|x-\frac{2}{\lambda}\right\| .
$$

Hence (2.1) holds. If $\lambda \operatorname{dist}\left(\frac{2}{\lambda}, T \frac{2}{\lambda}\right) \leq\left\|\frac{2}{\lambda}-x\right\|$, then $x \leq \frac{4}{\lambda(\lambda+2)}$ and

$$
H\left(T \frac{2}{\lambda}, T x\right)=a-\frac{x}{2} \leq \frac{2}{\lambda}-x=\left\|\frac{2}{\lambda}-x\right\| .
$$

Thus (2.2) holds. Therefore, $T$ satisfies condition $\left(C_{\lambda}\right)$. Clearly, $T$ is upper semicontinuous but not continuous (and hence $T$ is not nonexpansive). 
For a multivalued mapping $T: E \rightarrow C B(X)$, a sequence $\left\{x_{n}\right\}$ in $E$ of a Banach space $X$ for which $\lim _{n \rightarrow \infty} \operatorname{dist}\left(x_{n}, T x_{n}\right)=0$ is called an approximate fixed point sequence (afps for short) for $T$.

Let $(M, d)$ be a metric space. A geodesic path joining $x \in X$ to $y \in X$ is a map $c$ from a closed interval $[0, r] \subset \mathbb{R}$ to $X$ such that $c(0)=x, c(r)=y$ and $d(c(t), c(s))=|t-s|$ for all $s$, $t \in[0, r]$. The mapping $c$ is an isometry and $d(x, y)=r$. The image of $c$ is called a geodesic segment joining $x$ and $y$ which when unique is denoted by $\operatorname{seg}[x, y]$. A metric space $(M, d)$ is said to be of hyperbolic type if it is a metric space that contains a family $L$ of metric segments (isometric images of real line bounded segments) such that (a) each two points $x, y$ in $M$ are endpoints of exactly one member $\operatorname{seg}[x, y]$ of $L$, and (b) if $p, x, y$ $\in M$ and $m \in \operatorname{seg}[x, y]$ satisfies $d(x, m)=\alpha d(x, y)$ for $\alpha \in[0,1]$, then $d(p, m) \leq(1-\alpha) d$ $(p, x)+\alpha d(p, y) . M$ is said to be metrically convex if for any two points $x, y \in M$ with $x$ $\neq y$ there exists $z \in M, x \neq z \neq y$, such that $d(x, y)=d(x, z)+d(z, y)$. Obviously, every metric space of hyperbolic type is always metrically convex. The converse is true provided that the space is complete: If $(M, d)$ is a complete metric space and metrically convex, then $(M, d)$ is of hyperbolic type (cf. [[16], Page 24]). Clearly, every nonexpansive retract is of hyperbolic type.

Proposition 2.1. [[17], Proposition 2] Suppose (M, d) is of hyperbolic type, let $\left\{\alpha_{n}\right\} \subset$ $[0,1)$, if $\left\{x_{n}\right\}$ and $\left\{y_{n}\right\}$ are sequences in $M$ which satisfy for all $i, n$,

(i) $x_{n+1} \in \operatorname{seg}\left[x_{n}, y_{n}\right]$ with $d\left(x_{n}, x_{n+1}\right)=\alpha_{n} d\left(x_{n}, y_{n}\right)$,

(ii) $d\left(y_{n+1}, y_{n}\right) \leq d\left(x_{n+1}, x_{n}\right)$,

(iii) $d\left(y_{i+n}, x_{i}\right) \leq d<\infty$,

(iv) $\alpha_{n} \leq b<1$, and

(v) $\sum_{s=0}^{\infty} \alpha_{s}=+\infty$.

Then $\lim _{n \rightarrow \infty} d\left(y_{n}, x_{n}\right)=0$.

Let $E$ be a nonempty bounded closed subset of a Banach space $X$ and $\left\{x_{n}\right\}$ a bounded sequence in $X$. For $x \in X$, define the asymptotic radius of $\left\{x_{n}\right\}$ at $x$ as the number

$$
r\left(x,\left\{x_{n}\right\}\right)=\limsup _{n \rightarrow \infty}\left\|x_{n}-x\right\| .
$$

Let

$$
r\left(E,\left\{x_{n}\right\}\right)=\inf \left\{r\left(x,\left\{x_{n}\right\}\right): x \in E\right\}
$$

and

$$
A\left(E,\left\{x_{n}\right\}\right)=\left\{x \in E: r\left(x,\left\{x_{n}\right\}\right)=r\left(E,\left\{x_{n}\right\}\right)\right\} .
$$

The number $r\left(E,\left\{x_{n}\right\}\right)$ and the set $A\left(E,\left\{x_{n}\right\}\right)$ are, respectively, called the asymptotic radius and asymptotic center of $\left\{x_{n}\right\}$ relative to $E$. The sequence $\left\{x_{n}\right\}$ is called regular relative to $E$ if $r\left(E,\left\{x_{n}\right\}\right)=r\left(E,\left\{x_{n^{\prime}}\right\}\right)$ for each subsequence $\left\{x_{n^{\prime}}\right\}$ of $\left\{x_{n}\right\}$. It is well known that: every bounded sequence contains a subsequence that is regular relative to a given set (see [[16], Lemma 15.2] or [[18], Theorem 1]). Further, $\left\{x_{n}\right\}$ is called asymptotically uniform relative to $E$ if $A\left(E,\left\{x_{n}\right\}\right)=A\left(E,\left\{x_{n^{\prime}}\right\}\right)$ for each subsequence $\left\{x_{n^{\prime}}\right\}$ of $\left\{x_{n}\right\}$. It was noted in [16] that if $E$ is nonempty and weakly compact, then $A\left(E,\left\{x_{n}\right\}\right)$ is nonempty and weakly compact, and if $E$ is convex, then $A\left(E,\left\{x_{n}\right\}\right)$ is convex. 
A Banach space $X$ is said to satisfy the Kirk-Massa condition if the asymptotic center of each bounded sequence of $X$ in each bounded closed and convex subset is nonempty and compact. A more general space than spaces satisfying the Kirk-Massa condition is a space having property $(D)$. Property $(D)$ introduced in [19] is defined as follows:

Definition 2.2. [[19], Definition 3.1] A Banach space $X$ is said to have property $(D)$ if there exists $\lambda \in[0,1)$ such that for any nonempty weakly compact convex subset $E$ of $X$, any sequence $\left\{x_{n}\right\} \subset E$ that is regular and asymptotically uniform relative to $E$, and any sequence $\left\{y_{n}\right\} \subset A\left(E,\left\{x_{n}\right\}\right)$ that is regular and asymptotically uniform relative to $E$ we have

$$
r\left(E,\left\{y_{n}\right\}\right) \leq \lambda r\left(E,\left\{x_{n}\right\}\right) .
$$

Theorem 2.3. [[19], Theorem 3.6] Let E be a nonempty weakly compact convex subset of a Banach space $X$ that has property $(D)$. Assume that $T: E \rightarrow K C(E)$ is a nonexpansive mapping. Then, $T$ has a fixed point.

A direct consequence of Theorem 2.3 is that every weakly compact convex subset of a space having property $(D)$ has both (MFPP) for multivalued nonexpansive mappings and (CFPP). The class of spaces having property $(D)$ contains several well-known ones including $k$-uniformly rotund, nearly uniformly convex, uniformly convex in every direction spaces, and spaces satisfying Opial condition (see [3,19-23] and references therein).

The following useful result is due to Bruck:

Theorem 2.4. [[14], Theorem 1] Let E be a nonempty closed convex subset of a Banach space $X$. Suppose $E$ is locally weakly compact and $F$ is a nonempty subset of $E$. Let $N(F)=\{f \mid f\}: E \rightarrow E$ is nonexpansive and $f x=x$ for all $x \in F\}$. Suppose that for each $z$ in $E$, there exists $h$ in $N(F)$ such that $h(z) \in F$. Then, $F$ is a nonexpansive retract of $E$.

\section{Main results}

We first state three main results:

Theorem 3.1. Let E be a weakly compact convex subset of a Banach space X. Suppose $E$ has (MFPP) and (CFPP). Let $S$ be any commuting family of nonexpansive self-mappings of $E$. If $T: E \rightarrow K C(E)$ is a multivalued nonexpansive mapping that commutes with every member of $S$. Then, $F(S) \cap F i x(T) \neq \varnothing$.

Theorem 3.2. Let $X$ be a Banach space satisfying the Kirk-Massa condition and let $E$ be a weakly compact convex subset of $X$. Let $S$ be any commuting family of nonexpansive self-mappings of $E$. Suppose $T: E \rightarrow K C(E)$ is a multivalued mapping satisfying condition $\left(C_{\lambda}\right)$ for some $\lambda \in(0,1)$ that commutes with every member of $S$. If $T$ is upper semi-continuous, then $F(S) \cap F i x(T) \neq \varnothing$.

Theorem 3.3. Let E be a weakly compact convex subset of a Banach space X. Suppose $E$ has (MFPP) and (CFPP). Let $S$ be any commuting family of nonexpansive self-mappings of $E$. If $T: E \rightarrow K C(E)$ is a multivalued nonexpansive mapping that commutes with every member of $S$. Suppose in addition that $T$ satisfies.

(i) there exists a nonexpansive mapping $s: E \rightarrow E$ such that $s x \in T x$ for each $x \in E$,

(ii) $\operatorname{Fix}(T)=\{x \in E: T x=\{x\}\} \neq \varnothing$. 
Then, $F(S) \cap F i x(T)$ is a nonempty nonexpansive retract of $E$.

\section{Remark 3.4.}

(i) As corollaries, the results in Theorems 3.1 and 3.3 are valid for spaces $X$ having property $(D)$.

(ii) Theorem 3.3 can be viewed as a generalization of Theorem 1.4 of Bruck for weakly compact convex domains.

Definition 3.5. Let $E$ be a nonempty bounded closed and convex subset of a Banach space $X$. Let $t: E \rightarrow E$ be a single valued mapping, $T: E \rightarrow K C(E)$ a multivalued mapping. Then, $t$ and $T$ are said to be commuting mappings if $t T x \subset T$ Ttx for all $x \in E$.

If in Theorem 2.4, we put $F=\operatorname{Fix}(t)$ where $t: E \rightarrow E$ is nonexpansive, then it was noted in [[15], Remark 1] that a retraction $c \in N(F)$ can be chosen so that $c W \subset W$ for all $t$ invariant closed and convex subsets $W$ of $E$. With the same proof, we can show that the same result is valid for $F=F(S)$. In this case, we define $N(F(S))=\{f \mid f: E \rightarrow E$ is nonexpansive, $F i x(f) \supset F(S), f(W) \subset W$ whenever $W$ is a closed convex $S$-invariant subset of $E$ \}. Here, by an " $S$-invariant"subset, we mean a subset that is left invariant under every member of $S$.

Lemma 3.6. Let $E$ be a nonempty weakly compact convex subset of a Banach space $X$ and let $S$ be any commuting family of nonexpansive self-mappings of E. Suppose that $E$ has (FPP) and (CFPP). Then, $F(S)$ is a nonempty nonexpansive retract of $E$, and a retraction $c$ can be chosen so that every $S$-invariant closed and convex subset of $E$ is also c-invariant.

Proof. Note by Theorem 1.4 that $F(S)$ is nonempty. According to Theorem 2.4, it suffices to show that for each $z$ in $E$, there exists $h$ in $N(F(S))$ such that $h(z) \in F(S)$.

Let $z \in E$ and $K=\{f(z) \mid f \in N(F(S))\} \subset E$. Since $K$ is weakly compact convex and invariant under every member in $S$, we obtain by Theorem 1.4 that $F(S) \cap K \neq \varnothing$, i.e., there exists $h$ in $N(F(S))$ such that $h(z) \in F(S)$. Theorem 2.4 then implies that $F(S)$ is a nonexpansive retract of $E$, where a retraction is chosen from $N(F(S))$.

Proof of Theorem 3.1 Let $c$ be a nonexpansive retraction of $E$ onto $F(S)$ obtained in Lemma 3.6. Set $U x:=T c x$ for $x \in E$. Clearly,

$$
H(U x, U y)=H(T c x, T c y) \leq\|c x-c y\| \leq\|x-y\| \text { for } x, y \in E .
$$

Thus, $U$ is nonexpansive, and since $E$ has (MFPP), there exists $p \in U p=T c p$. Since $T c p$ is $S$-invariant, by the property of $c, T c p$ is also $c$-invariant, i.e., $c p \in T c p$. Therefore, $F(S) \cap F i x(T) \neq \varnothing$. $\square$

The following proposition is needed for a proof of Theorem 3.2.

Proposition 3.7. Let $A$ be a compact convex subset of a Banach space $X$ and let $a$ nonempty subset $F$ of $A$ be a nonexpansive retract of $A$. Suppose a mapping $U: A \rightarrow$ $K C(A)$ is upper semi-continuous and satisfies:

(i) $c(U x) \subset U x$ for all $x \in F$ where $c$ is a nonexpansive retraction of $A$ onto $F$, and

(ii) $F$ is $U$-invariant.

Then, $U$ has a fixed point in $F$. 
Proof. Let $\varepsilon>0$. Since $F$ is compact, there exists a finite $\varepsilon$-dense subset $\left\{z_{1}, z_{2}, \ldots, z_{n}\right\}$ of $F$, i.e., $F \subset \bigcup_{i=1}^{n} B\left(z_{i}, \frac{\varepsilon}{2}\right)$. Put $K:=\overline{c o}\left(z_{1}, z_{2}, \ldots, z_{n}\right)$ and define $V x=\bar{B}_{\varepsilon}(U c x) \cap K$ for $x \in K$. Clearly, $V: K \rightarrow K C(K)$. For $x \in K, c x \in F$ thus by (ii) there exists $y \in U c x \cap$ $F$. Then, choose $z_{i}$ for some $i$ such that $\left\|z_{i}-y\right\| \leq \frac{\varepsilon}{2}$. Therefore, $z_{i} \in \bar{B}_{\varepsilon}(U c x) \cap K$, i.e., $V x$ is nonempty for $x \in K$. We now show that $V$ is upper semi-continuous. Let $\left\{x_{n}\right\}$ be a sequence in $K$ converging to some $x \in K$ and $y_{n} \in V x_{n}$ with $y_{n} \rightarrow y$. Choose $a_{n}$ $\in U c x_{n}$ such that $\left\|y_{n}-a_{n}\right\| \leq \varepsilon$. As $A$ is compact, we may assume that $a_{n} \rightarrow a$ for some $a \in A$. By upper semi-continuity of $U, a \in U c x$. Consider

$$
\|y-a\| \leq\left\|y-\gamma_{n}\right\|+\left\|y_{n}-a_{n}\right\|+\left\|a_{n}-a\right\| .
$$

By letting $n \rightarrow \infty$, we obtain $\|y-a\| \leq \varepsilon$, i.e., $y \in V x$ and the proof that $V$ is upper semi-continuous is complete. By Kakutani fixed point theorem, there exists $p_{\varepsilon} \in V p_{\varepsilon}$, that is, $\left\|p_{\varepsilon}-b_{\varepsilon}\right\| \leq \varepsilon$ for some $b_{\varepsilon} \in U c p_{\varepsilon}$.

By the assumption on $U$, we see that $c b_{\varepsilon} \in U c p_{\varepsilon}$ and $\left\|c p_{\varepsilon}-c b_{\varepsilon}\right\| \leq|| p_{\varepsilon}-b_{\varepsilon} \| \leq \varepsilon$. Taking $\varepsilon=\frac{1}{n}$ and write $q_{n}$ for $c p_{\frac{1}{n}}$ and $b_{n}$ for $c b_{\frac{1}{n}}$, we obtain a sequence $\left\{q_{n}\right\} \subset F$ and $b_{n}$ $\in U q_{n} \cap F$ with $\left\|q_{n}-b_{n}\right\| \rightarrow 0$. By the compactness of $F$, we assume that $q_{n} \rightarrow q$ and $b_{n}$ $\rightarrow b$. It is seen that $q=b \in U q$.

Proof of Theorem 3.2 As observed earlier, $E$ has both (FPP) and (CFPP), thus we start with a nonexpansive retraction $c$ of $E$ onto $F(S)$ obtained by Lemma 3.6. For each $x \in F(S), T x$ is invariant under every member of $S$ and $T x$ is convex, thus $T x$ is $c$ invariant. Clearly, $c$ is a nonexpansive retraction of $T x$ onto $T x \cap F(S)$ that is nonempty by Theorem 1.4.

Next, we show that there exists an afps for $T$ in $F(S)$. Let $x_{0} \in F(S)$. Since $T x_{0} \cap F$ $(S) \neq \varnothing$, we can choose $y_{0} \in T x_{0} \cap F(S)$. Since $F(S)$ is of hyperbolic type, there exists $x_{1} \in F(S)$ such that

$$
\left\|x_{0}-x_{1}\right\|=\lambda\left\|x_{0}-y_{0}\right\| \text { and }\left\|x_{1}-y_{0}\right\|=(1-\lambda)\left\|x_{0}-y_{0}\right\| .
$$

Choose $y_{1}^{\prime} \in T x_{1}$ for which $\left\|y_{o}-y_{1}^{\prime}\right\|=\operatorname{dist}\left(y_{0}, T x_{1}\right)$. Set $y_{1}=c y_{1}^{\prime}$. Clearly, $\left\|y_{0}-y_{1}\right\|$ $=\left\|c y_{0}-c y_{1}^{\prime}\right\| \leq\left\|y_{0}-y_{1}^{\prime}{ }_{1}\right\|$. Therefore, we can choose $y_{1} \in T x_{1} \cap F(S)$ so that $\| y_{0}-$ $y_{1} \|=\operatorname{dist}\left(y_{0}, T x_{1}\right)$. In this way, we will find a sequence $\left\{x_{n}\right\} \subset F(S)$ satisfying

$$
\left\|x_{n}-x_{n+1}\right\|=\lambda|| x_{n}-y_{n} \| \text { and }\left\|x_{n+1}-y_{n}\right\|=(1-\lambda)|| x_{n}-y_{n} \|,
$$

where $y_{n} \in T x_{n} \cap F(S)$ and $\left\|y_{n}-y_{n+1}\right\|=\operatorname{dist}\left(y_{n}, T x_{n+1}\right)$.

Since $\lambda \operatorname{dist}\left(x_{n}, T x_{n}\right) \leq \lambda|| x_{n}-y_{n}\|=\| x_{n}-x_{n+1} \|$,

$$
\left\|y_{n}-y_{n+1}\right\| \leq H\left(T x_{n}, T x_{n+1}\right) \leq\left\|x_{n}-x_{n+1}\right\| .
$$

From Proposition 2.1, $\lim _{n \rightarrow \infty}|| y_{n}-x_{n} \|=0$ and $\left\{x_{n}\right\}$ is an afps for $T$ in $F(S)$. Assume that $\left\{x_{n}\right\}$ is regular relative to $E$. By the Kirk-Massa condition, $A:=A\left(E,\left\{x_{n}\right\}\right)$ is assumed to be nonempty compact and convex. Define $U x=T x \cap A$ for $x \in A$. We are going to show that $U x$ is nonempty for each $x \in A$. First, let $r:=r\left(E\right.$, \{ $\left.\left.x_{n}\right\}\right)$. If $r=0$ and if $x \in A$, then $x_{n} \rightarrow x$ and $y_{n} \rightarrow x$. Using upper semi-continuity of $T$, we see that $x \in T x$, i.e., $F(S) \cap \operatorname{Fix}(T) \neq \varnothing$.

Therefore, we assume for the rest of the proof that $r>0$. Let $x \in A$. If for some subsequence $\left\{x_{n_{k}}\right\}$ of $\left\{x_{n}\right\}, \lambda \operatorname{dist}\left(x_{n_{k}}, T x_{n_{k}}\right) \geq\left\|x_{n_{k}}-x\right\|$ for each $k$, we have 


$$
0=\limsup _{n \rightarrow \infty} \lambda \operatorname{dist}\left(x_{n_{k}}, T x_{n_{k}}\right) \geq \limsup _{n \rightarrow \infty}\left\|x_{n_{k}}-x\right\| \geq r
$$

since $\left\{x_{n}\right\}$ is regular relative to $E$ and this is a contradiction. Therefore,

$$
\lambda \operatorname{dist}\left(x_{n}, T x_{n}\right) \leq\left\|x_{n}-x\right\| \text { for sufficiently large } n \text {. }
$$

Now, we show that $U x$ is nonempty. Choose $y_{n} \in T x_{n}$ so that $\left\|x_{n}-y_{n}\right\|=\operatorname{dist}\left(x_{n}\right.$, $\left.T x_{n}\right)$ and choose $z_{n} \in T x$ such that $\left\|y_{n}-z_{n}\right\|=\operatorname{dist}\left(y_{n}, T x\right)$. As $T x$ is compact, we may assume that $\left\{z_{n}\right\}$ converges to $z \in T x$. Using (3.1) and the fact that $T$ satisfies condition $\left(C_{\lambda}\right)$, we have

$$
\begin{aligned}
\left\|x_{n}-z\right\| & \leq\left\|x_{n}-y_{n}\right\|+\left\|y_{n}-z_{n}\right\|+\left\|z_{n}-z\right\| \\
& =\left\|x_{n}-y_{n}\right\|+\operatorname{dist}\left(y_{n}, T x\right)+\left\|z_{n}-z\right\| \\
& \leq\left\|x_{n}-y_{n}\right\|+H\left(T x_{n}, T x\right)+\left\|z_{n}-z\right\| \\
& \leq\left\|x_{n}-y_{n}\right\|+\left\|x_{n}-x\right\|+\left\|z_{n}-z\right\| \text { for sufficiently large } n .
\end{aligned}
$$

Taking lim $\sup _{n \rightarrow \infty}$ in the above inequalities to obtain

$$
\limsup _{n \rightarrow \infty}\left\|x_{n}-z\right\| \leq \limsup _{n \rightarrow \infty}\left\|x_{n}-x\right\|=r
$$

that implies that $z \in U x$ proving that $U x$ is nonempty as claimed.

Now, we show that $U$ is upper semi-continuous. Let $\left\{z_{k}\right\}$ be a sequence in $A$ converging to some $z \in A$ and $y_{k} \in U z_{k}$ with $y_{k} \rightarrow y$. Consider the following estimates:

$$
\limsup _{n \rightarrow \infty}\left\|x_{n}-\gamma\right\| \leq \limsup _{n \rightarrow \infty}\left\|x_{n}-y_{k}\right\|+\limsup _{n \rightarrow \infty}\left\|y_{k}-y\right\|=r\left(E,\left\{x_{n}\right\}\right)+\limsup _{n \rightarrow \infty}\left\|y_{k}-\gamma\right\| \text { for each } k \text {. }
$$

Letting $k \rightarrow \infty$, it follows that

$$
\limsup _{n \rightarrow \infty}\left\|x_{n}-y\right\| \leq r\left(E_{,}\left\{x_{n}\right\}\right) .
$$

Hence $y \in A$. From upper semi-continuity of $T, y \in T z$. Therefore, $y \in U z$ and thus $U$ is upper semi-continuous. Put $F:=F(S) \cap A$. Since $A$ is $c$-invariant, it is clear that $F$ is a nonexpansive retract of $A$ by the retraction $c$. Now, if $x \in F$, then $U x$ is $S$-invariant which implies $U x$ is $c$-invariant. Therefore, condition (i) in Proposition 3.7 is justified. To verify condition (ii), we let $x \in F$. Take $y \in U x$. It is obvious that $c y \in U x \cap F(S)$, so $U$ satisfies condition (ii) of Proposition 3.7. Upon applying Proposition 3.7 we obtain a fixed point in $F$ of $U$ and thus of $T$ and we are done.

Proof of Theorem 3.3 By $(i)$ and $(i i)$, it is seen that Fix $(T)=\operatorname{Fix}(s)$. Note by Theorem 3.1 that $F(S) \cap F i x(s)$ is nonempty. Let $c$ be a retraction from $E$ onto $F(S)$ obtained by Lemma 3.6. Here, $c$ belongs to the set $N(F(S))=\{f \mid f: E \rightarrow E$ is nonexpansive, Fix (f) $\supset F(S), f(W) \cap W$ whenever $W$ is a closed convex $S$-invariant subset of $E$ ). Put $F=F$ $(S) \cap \operatorname{Fix}(s)$ and let $N(F)=\{f \mid f: E \rightarrow E$ is nonexpansive, $F i x(f) \supset F\}$. Let $z \in E$ and consider the weakly compact and convex set $K:=\{f(z) \mid f \in N(F)\}$. It is left to show that $h(z) \in F$ for some $h \in N(F)$. Since $K$ is $S$-invariant, $K$ is therefore $c$-invariant. It is evident that $K$ is $s$-invariant. Thus $s c: K \rightarrow K$. Therefore, $s c$ has a fixed point, say $x$, in $K$, i.e., $s c(x)=x$. By $(i), s c(x) \in T c x$. Since $T c x$ is $c$-invariant, we have $c x \in T c x$. That is $c x$ $\in \operatorname{Fix}(T)=\operatorname{Fix}(s)$. Hence $s c x=x=c x$, i.e., $c x \in F(S) \cap F i x(s)$, and the proof is complete.

When $S$ consists of only the identity mapping of $E$, we immediately have the following corollary: 
Corollary 3.8. Let E be a weakly compact convex subset of a Banach space X. Suppose $E$ has (MFPP). If $T: E \rightarrow K C(E)$ is a multivalued nonexpansive mapping satisfying.

(i) there exists a nonexpansive mapping $s: E \rightarrow E$ such that $s x \in T x$ for each $x \in E$,

(ii) $\operatorname{Fix}(T)=\{x \in E: T x=\{x\}\} \neq \varnothing$.

Then Fix $(T)$ is a nonempty nonexpansive retract of $E$.

Of course, when $T$ is single valued, condition $(i)$ is satisfied. Even a very simple example shows that condition (ii) in Corollary 3.8 may not be dropped.

Example 3.9. Let $X$ be the Hilbert space $\mathbb{R}^{2}$ with the usual norm, and let $f:[0,1] \rightarrow$ $[0,1]$ be a continuous function that is strictly concave, $f(0)=\frac{1}{2}$ and $f(1)=1$. Moreover let $f^{\prime}(x) \leq 1$ for $x \in[0,1]$. Let $T:[0,1]^{2} \rightarrow K C\left([0,1]^{2}\right)$ be defined by $T(x, y)=[0, x] \times$ $[f(x), 1]$. We show that $T$ is nonexpansive, but $\operatorname{Fix}(T) \neq\{x: T x=\{x\}\}$ and $\operatorname{Fix}(T)$ is not metrically convex. If $\left(x_{1}, y_{1}\right),\left(x_{2}, y_{2}\right) \in[0,1]^{2}$, then

$$
H\left(T\left(x_{1}, y_{1}\right), T\left(x_{2}, y_{2}\right)\right)=\left|x_{1}-x_{2}\right| \leq\left\|\left(x_{1}, y_{1}\right)-\left(x_{2}, y_{2}\right)\right\| .
$$

Hence $T$ is nonexpansive. However, $a=\left(0, \frac{1}{2}\right)$ is a fixed point but $T a \neq\{a\}$. Finally, Fix (T) is not metrically convex since, putting $b=(1,1)$, we see that $b \in T b$, but $\frac{a+b}{2}=\left(\frac{1}{2}, \frac{3}{4}\right) \notin T \frac{a+b}{2}$ since $f$ is strictly concave.

In [[14], Lemma 6] it was stated that: Let $E$ be a nonempty weakly compact convex subset of a Banach space $X$. Suppose $E$ has (HFPP). Suppose $F$ is a nonempty nonexpansive retract of $E$ and $t: E \rightarrow E$ is a nonexpansive mapping which leaves $F$ invariant. Then $F i x(t) \cap F$ is a nonempty nonexpansive retract of $E$.

Here, we have a multivalued version (with a similar proof) of this result.

Corollary 3.10. Let $E$ and $T$ be as in Corollary 3.8. Suppose $F$ is a nonexpansive retract of $E$ by a retraction c. If $T x$ is c-invariant for each $x \in F$, then $F i x(T) \cap F$ is a nonempty nonexpansive retract of $E$.

\section{Acknowledgements}

The authors are grateful to the referees for their valuable comments. They also wish to thank the National Research University Project under Thailand's Office of the Higher Education Commission for financial support.

Author details

${ }^{1}$ Department of Mathematics, Faculty of Science, Chiang Mai University, Chiang Mai 50200, Thailand ${ }^{2}$ Materials Science Research Center, Faculty of Science, Chiang Mai University, Chiang Mai 50200, Thailand

\section{Authors' contributions}

All authors read and approved the final manuscript.

\section{Competing interests}

The authors declare that they have no competing interests.

Received: 4 May 2011 Accepted: 17 September 2011 Published: 17 September 2011

References

1. Dhompongsa, S, Khaewkhao, A, Panyanak, B: Lim's theorems for multivalued mappings in CAT(0) spaces. J Math Anal Appl. 312, 478-487 (2005). doi:10.1016/j.jmaa.2005.03.055

2. Shahzad, N, Markin, J: Invariant approximations for commuting mappings in CAT(0) and hyperconvex spaces. J Math Anal Appl. 337 (2008)

3. Dhompongsa, S, Khaewcharoen, A, Khaewkhao, A: The Domínguez-Lorenzo condition and fixed points for multivalued mappings. Nonlinear Anal. 64, 958-970 (2006). doi:10.1016/j.na.2005.05.051

4. Abkar, A, Eslamian, M: Fixed point theorems for Suzuki generalized nonexpansive multivalued mappings in Banach spaces. Fixed Point Theory Appl 1-10 (2010). Article ID 457935

5. Abkar, A, Eslamian, M: Common fixed point results in CAT(0) spaces. Nonlinear Anal. 74, 1835-1840 (2011). doi:10.1016/j. na.2010.10.056 
6. Espínola, R, Hussain, N: Common fixed points for multimaps in metric spaces. Fixed Point Theory Appl 1-14 (2010). Article ID 204981

7. Espínola, R, Fernández-León, A, Piatek, B: Fixed points of single- and set-valued mappings in uniformly convex metric spaces with no metric convexity. Fixed Point Theory Appl 1-16 (2010). Article ID 169837

8. Espínola, R, Lorenzo, P, Nicolae, A: Fixed points, selections and common fixed points for nonexpansive-type mappings. $J$ Math Anal Appl. 382, 503-515 (2011). doi:10.1016/j.jmaa.2010.06.039

9. Hussain, N, Khamsi, MA: On asymptotic pointwise contractions in metric spaces. Nonlinear Anal. 71, 4423-4429 (2009). doi:10.1016/j.na.2009.02.126

10. Khaewcharoen, A, Panyanak, B: Fixed points for multivalued mappings in uniformly convex metric spaces. Int J Math Math Sci 1-9 (2008). Article ID 163580

11. Razani, A, Salahifard, H: Invariant approximation for CAT(0) spaces. Nonlinear Anal. 72, 2421-2425 (2010). doi:10.1016/j. na.2009.10.039

12. Shahzad, N: Fixed point results for multimaps in CAT(0) spaces. Topol Appl. 156, 997-1001 (2009). doi:10.1016/j. topol.2008.11.016

13. Shahzad, N: Invariant approximations in CAT(0) spaces. Nonlinear Anal. 70, 4338-4340 (2009). doi:10.1016/j. na.2008.10.002

14. Bruck, RE Jr: Properties of fixed-point sets of nonexpansive mappings in Banach spaces. Trans Am Math Soc. 179, 251-262 (1973)

15. Bruck, RE Jr: A common fixed point theorem for a commuting family of nonexpansive mappings. Pac J Math. 53, 59-71 (1974)

16. Goebel, K, Kirk, WA: Topics in Metric Fixed Point Theory. Cambridge University Press, Cambrige (1990)

17. Goebel, K, Kirk, WA: Iteration processes for nonexpansive mappings. Contemp Math. 21 (1983)

18. Lim, TC: A fixed point theorem for multivalued nonexpansive mappings in a uniformly convex Banach space. Bull Am Math Soc. 80, 1123-1126 (1974). doi:10.1090/50002-9904-1974-13640-2

19. Dhompongsa, S, Domínguez Benavides, T, Khaewcharoen, A, Khaewkhao, A, Panyanak, B: The Jordan-von Neumann constants and fixed points for multivalued nonexpansive mappings. J Math Anal Appl. 320, 916-927 (2006). doi:10.1016/j.jmaa.2005.07.063

20. Domínguez Benavides, T, Gavira, B: The fixed point property for multivalued nonexpansive mappings. J Math Anal Appl. 328, 1471-1483 (2007). doi:10.1016/j.jmaa.2006.06.059

21. Domínguez Benavides, T, Lorenzo Ramírez, P: Fixed point theorems for multivalued nonexpansive mappings without uniform convexity. Abstr Appl Anal. 6, 375-386 (2003)

22. Kaewkhao, A: The James constant, the Jordan von Neumann constant, weak orthogonality, and fixed points for multivalued mappings. J Math Anal Appl. 333, 950-958 (2007). doi:10.1016/j.jmaa.2006.12.001

23. Saejung, S: Remarks on sufficient conditions for fixed points of multivalued nonexpansive mappings. Nonlinear Anal. 67, 1649-1653 (2007). doi:10.1016/j.na.2006.07.037

doi:10.1186/1687-1812-2011-54

Cite this article as: Nanan and Dhompongsa: A common fixed point theorem for a commuting family of nonexpansive mappings one of which is multivalued. Fixed Point Theory and Applications 2011 2011:54

\section{Submit your manuscript to a SpringerOpen ${ }^{\circ}$ journal and benefit from:}

- Convenient online submission

- Rigorous peer review

- Immediate publication on acceptance

- Open access: articles freely available online

- High visibility within the field

- Retaining the copyright to your article

Submit your next manuscript at $\gg$ springeropen.com 